\section{Brain, Behavior and Evolution}

Brain Behav Evol 2016;87:65-68

DOI: $10.1159 / 000443716$
Published online: February 12, 2016

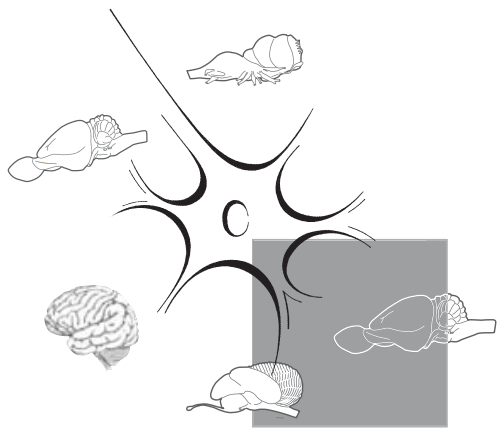

\title{
Sleep, Evolution and Brains
}

\author{
Commentary on Herculano-Houzel S (2015): Decreasing Sleep \\ Requirement with Increasing Numbers of Neurons as a Driver for Bigger \\ Brains and Bodies in Mammalian Evolution. Proc Biol Sci 282:20151853
}

\author{
Robert A. Barton ${ }^{a}$ Isabella Capellini ${ }^{b}$ \\ aEvolutionary Anthropology Research Group, Department of Anthropology, Durham \\ University, Durham, and bSchool of Biological, Biomedical and Environmental Sciences, \\ University of Hull, Hull, UK
}

Why do we sleep? In a sense, it is equally valid to ask why we wake, except that the functions of the things we do while awake - eating and reproducing, for example are quite obvious, whereas the same is not true for the things that we do while asleep - such as rapid eye movements and dreaming. This is why the function of sleep has exercised the minds of scientists, writers and philosophers since Aristotle. Sleep appears to be biologically necessary: sleep deprivation in mammals is followed by a tiredness-induced 'rebound' and, if chronic, leads to impaired physiological and mental functioning, or, in the extreme, even death [Allada and Siegel, 2008]. Yet despite its apparent necessity, a consensus on the function of sleep remains elusive: prominent hypotheses include conservation of energy when activities are unprofitable or impossible [Siegel, 2009], memory consolidation [Stickgold and Walker, 2007] or some physiological function, such as maintenance of the immune system [Opp and Krueger, 2015]. So many different hypotheses have been proposed that some have even suggested that sleep has no specific biological function [Rial et al., 2007], although, as Rechtschaffen [1971] asserted, in that case sleep must be 'the biggest mistake the evolutionary process ever made'.

\section{KARGER}

(c) 2016 S. Karger AG, Basel

0006-8977/16/0872-0065\$39.50/0

E-Mail karger@karger.com

www.karger.com/bbe
Much research points to important and diverse effects of sleep on neural and cognitive functioning, suggesting that the relationship between sleep and the brain holds the key [Hobson, 2005]. Sleep is associated with distinctive patterns of neural activity which differ between rapid-eye movement (REM) sleep and non-REM sleep [Staunton, 2005]. Sleep is thought to play a role in synaptic remodelling [Jha et al., 2005], and sleep deprivation negatively impacts on a variety of aspects of cognitive function, including learning, reaction times and attention [Hobson, 2005; Staunton, 2005].

The biology and functions of sleep have mostly been tackled using experiments. By carefully manipulating certain variables and holding others constant, researchers have attempted to isolate effects that are specific to sleep and to particular aspects of sleep (notably REM vs. nonREM). Complementing experimental approaches are cross-species comparisons, which potentially provide important evidence about biological function and evolution.

It has been known for many years that the amount of time spent sleeping is highly variable across mammal species, from about $3 \mathrm{~h}$ to about $20 \mathrm{~h}$ per day [Siegel, 2009]. In principle, any hypothesis for the function of sleep should be capable of explaining this variation, but pin- 
ning down robust correlates has not proved easy [Lesku et al., 2006; Capellini et al., 2008a]. In mammals, smaller species tend to sleep more than larger species and are more likely to be polyphasic, dividing their 24-hour sleep quota between two or more bouts [Capellini et al., 2008b; Siegel 2009]. The posited neural functions of sleep have been tested by examining correlations between sleep and brain size or the size of specific brain structures, but few robust correlates across mammals and birds have emerged [Lesku et al, 2006; Roth et al., 2006; Capellini et al., 2008a, 2009]. In cetaceans, non-REM sleep occurs unihemispherically and REM sleep is dramatically reduced, but the biological significance of this difference with land mammals is unclear [Pillay and Manger, 2004].

One idea that has gained increasing traction is that the variety of the observed cognitive effects of sleep deprivation may be explained by some general neurophysiological housekeeping function, such as the removal of toxic metabolites that build up in the brain during wakeful activity and which are likely to damage neurons. A pertinent observation is that proteins linked to neurodegenerative diseases, such as $\beta$-amyloid, accumulate in the interstitial space surrounding cells of the brain, and these proteins are removed by convective exchange between interstitial fluid (ISF) and cerebrospinal fluid (CSF) [Xie et al., 2013]. There is now converging evidence for this hypothesis from experimental and comparative studies. Xie et al. [2013] used in vivo imaging to compare CSF influx in awake, anaesthetised and asleep mice. They found that interstitial space increases markedly during natural sleep and anaesthesia, causing an increase in the convective exchange of CSF and ISF, and an increase in the rate of $\beta$-amyloid clearance.

Now, Herculano-Houzel [2015] has extended the hypothesis of Xie et al. [2013] to try and explain the evolution of variation in sleep times. Using comparative data on sleep durations and brain composition in 24 mammalian species, Herculano-Houzel [2015] finds that total sleep time (TST) correlates with several neuro-anatomical variables, including cortical neuron density, thickness and surface area. The strongest correlation was between sleep time and the ratio of cortical neuron density to surface area (D/A). Herculano-Houzel [2015] argues that this strong positive correlation of sleep with D/A is consistent with the hypothesis of Xie et al. [2013], because metabolites should accumulate faster if there is a high density of neurons relative to the surface area for perfusion by CSF. The verbal model of Herculano-Houzel [2015] makes certain assumptions, for example concerning the dynamics of the exchange between CSF and ISF and the relationships among neuron density, volume, surface area and metabolite accumulation. These assumptions will need further examination, but they are a useful starting point.

As the readers of this journal are well aware, comparative analysis is a powerful tool for studying evolutionary questions, such as how sleep and brains co-evolved. As with any form of analysis, there are potential pitfalls, and it can be tricky to disentangle the web of relationships among putatively important and confounding variables, particularly where these variables correlate with overall body and/or brain size. Herculano-Houzel [2015] recognised this problem, and conducted a principal components analysis that identified two factors; a general size factor and a second factor comprising D/A, cortical thickness and sleep time, lending weight to the claim that the correlations observed are explained by specific neural mechanisms rather than by other confounds or allometric factors.

Two other potential issues in comparative studies that may affect our ability to identify genuine statistical relationships are phylogenetic non-independence among data points and data quality. Phylogenetic effects need to be carefully considered in any comparative study: similarities between species reflect the recency of common ancestry as well as independent evolution, and ignoring phylogeny therefore violates statistical assumptions and can give misleading results [Nunn, 2011]. Data quality is also critical. Ideally, sleep data should be recorded in individuals of uniform age (since sleep quotas decrease markedly during development), and over at least a 12hour cycle under strictly regulated conditions. In addition, purely behavioural assessment rather than EEG overestimates sleep quotas, so mixing the two kinds of data is problematic [Capellini et al., 2008a] and use of EEG-based measures of sleep quotas is recommended [McNamara et al., 2008]. Some of the data used by Herculano-Houzel [2015] do not reach this high standard. For example, the data point for Cebus apella was based on behavioural rather than EEG recordings of a single infant for less than $12 \mathrm{~h}$, so is not reliable as an estimate of adult sleep time in this species.

What happens if we re-examine the relationships between sleep times and brain composition taking the aforementioned issues into account? We analysed the sleep data used in Capellini et al. [2008a, b], which were based on the above criteria (data from adults recorded using EEG over at least $12 \mathrm{~h}$ ); this increase in data quality is traded off against a smaller sample size, with the number of species reduced from 24 to 19 . We analysed the logged
66

Brain Behav Evol 2016;87:65-68 DOI: $10.1159 / 000443716$
Barton/Capellini 
data using phylogenetic generalised least squares (PGLS) implemented by the Caper program in R [Orme et al., 2015]. We used a phylogeny derived from a mammal supertree with updated branch lengths [Bininda-Emonds et al., 2007, 2008] widely used in comparative studies, pruned to match the species in the data. In PGLS, the phylogenetic signal is accounted for and estimated as the parameter $\lambda$ [Freckleton et al., 2002]. The good news is that this phylogenetic analysis on a reduced but higher quality sleep data set confirms the positive correlation between TST and D/A $\left(\mathrm{t}_{1,17}=2.70, \mathrm{p}=0.015, \lambda=0.85\right.$, adjusted $\left.\mathrm{r}^{2}=0.26\right)$, although the relationship is weaker than in Herculano-Houzel's analysis.

Less encouraging is the finding that there is a slightly stronger correlation between TST and body size $\left(\mathrm{t}_{1,17}=\right.$ $2.96, \mathrm{p}=0.009, \lambda=0.73$, adjusted $\left.\mathrm{r}^{2}=0.30\right)$. The problem is that $\mathrm{D} / \mathrm{A}$ is strongly related to body size $\left(\mathrm{t}_{1,17}=10.79\right.$, $\mathrm{p}<0.0001, \lambda=0.21$, adjusted $\mathrm{r}^{2}=0.87$ ). If it is correct that the ratio D/A is a significantly stronger predictor of TST than general allometric factors, then it should still correlate with TST after accounting for the effects of body size, yet it does not $\left(t_{2,16}=0.51, p=0.62, \lambda=0.78\right.$, based on a multiple PGLS regression). This result does not demonstrate that Herculano-Houzel's conclusions are incorrect; as she points out, D/A could be intrinsically related to body size and still be the key driver of sleep time, such that variation in $\mathrm{D} / \mathrm{A}$ explains why large animals tend to sleep less. However, since brain size, neuro-anatomy and body size do vary to some extent independently of one another [e.g. Montgomery et al., 2010], it should theoretically be possible to tease apart their effects on sleep time.

Given that other explanations for the correlation of TST with body size have been proposed, such as the different trophic levels of larger versus smaller species [Capellini et al., 2008a; Siegel, 2009], more work will be required to definitively differentiate between the hypotheses. More high-quality data yielding greater statistical power would help in this endeavour, as would experi- mental and developmental approaches. Herculano-Houzel's study is likely to stimulate such work, and she has already made a start in that direction - in the same paper, she shows that the decline in sleep from birth onwards is associated with the predicted increase in the $\mathrm{D} / \mathrm{A}$ ratio (in rats). Again, however, more work is needed to differentiate between hypotheses for this developmental trend in sleep, since other explanations have been proposed for it [Siegel, 2005].

Herculano-Houzel [2015] also addresses the question of human sleep, suggesting that, together with other primates, more sleep seems to be required relative to the number of cortical neurons. She suggests that this could be due to the different scaling rules for cortical neuron density in primates compared to non-primates. In another new comparative study, however, Sampson and Nunn [2015] argue that humans have evolved to need significantly less sleep than their non-human primate relatives. They observe that the human sleep quota is the shortest recorded among primates', and they use a phylogenetic outlier test to determine that the human value falls significantly outside the expected range. They also observe that the reduced sleep time is associated with a significantly higher ratio of REM to non-REM sleep in humans. According to Sampson and Nunn [2015], lying down to sleep securely on a platform, nest or on the ground - a behaviour that evolved in the common ancestor of all great apes - may have been the trigger for the evolution of 'deeper' and more efficient sleep, enabling humans ultimately to reduce time spent asleep and thereby increase time available for other activities. Perhaps the increased intensity of sleep in humans reflects an enhancement of the physiological mechanism for clearing metabolites and permitting the benefits of sleep to be realised in a shorter time. Whatever the explanation for the observed variations across species in sleep time and sleep states, it is bound to attract further attention from evolutionary neurobiologists.

\section{References}

\footnotetext{
Allada R, Siegel JM (2008): Unearthing the phylo- Capellini I, Barton RA, Nunn CL, McNamara P genetic roots of sleep. Curr Biol 18:R670- (2008a): Phylogenetic analysis of the ecology R679.

Bininda-Emonds ORP, et al (2007): The delayed rise of present-day mammals. Nature 446: 507-512.

Bininda-Emonds ORP, et al (2008): The delayed rise of present-day mammals. Corrigendum. and evolution of mammalian sleep. Evolution 62:1764-1776.

Capellini I, Nunn CL, McNamara, P, Preston BT, Barton RA (2008b): Energetic constraints, not predation, influence the evolution of sleep patterning in mammals. Funct Ecol 22:847-853.

Capellini I, McNamara P, Preston BT, Nunn CL, reckleton RP, Harvey PH, Pagel MD (2002): Barton RA (2009): Does sleep play a role in memory consolidation? A comparative test. PLoS One 4:e4609. Phylogenetic analysis and comparative data: a test and review of evidence. Am Nat 160:712726
} 
-Jha SK, Jones, BE, Coleman T, Steinmetz N, Law C-T, Griffin G, Hawk J, Dabbish N, Kalatsky VA, Frank MG (2005): Sleep-dependent plasticity requires cortical activity. J Neurosci 25 : 9266-9274.

Herculano-Houzel S (2015): Decreasing sleep requirement with increasing numbers of neurons as a driver for bigger brains and bodies in mammalian evolution. Proc Biol Sci 282: 20151853.

Hobson JA (2005): Sleep is of the brain, by the brain and for the brain. Nature 437:12541256.

Lesku JA, Roth TC, Amlaner CJ, Lima SL (2006): A phylogenetic analysis of sleep architecture in mammals: the integration of anatomy, physiology, and ecology. Am Nat 168:441453.

- McNamara P, Capellini I, Harris E, Nunn CL, Barton RA, Preston B (2008): The phylogeny of sleep database: a new resource for sleep scientists. Open Sleep J 1:11-14.
Montgomery SH, Capellini I, Barton RA, Mundy NI (2010): Reconstructing the ups and downs of primate brain evolution: implications for adaptive hypotheses and Homo floresiensis. BMC Biol 27:9.

Nunn CL (2011): The Comparative Approach in Evolutionary Anthropology and Biology. Chicago, University of Chicago Press.

Opp MR, Krueger JM (2015): Sleep and immunity: a growing field with clinical impact. Brain Behav Immun 47:1-3.

Orme D, Freckleton R, Thomas G, Petzoldt T, Fritz S, Isaac N, Pearse W (2013): Comparative analyses of phylogenetics and evolution in R. https://cran.r-project.org/web/packages/caper/caper.pdf.

Pillay P, Manger P (2004): Testing thermogenesis as the basis for the evolution of cetacean sleep phenomenology. J Sleep Res 13:353-358.
Rechtschaffen A (1971): The control of sleep; in Hunt WA (ed): Human Behaviour and Its Control. Cambridge, Schenkman, pp 75-92.

- Rial RV, Nicolau MC, Gamundi A, Akaarir M, Aparicio S, Garau C, et al (2007): The trivial function of sleep. Sleep Med Rev 11:311-25.

Sampson DR, Nunn CL (2015): Sleep intensity and the evolution of human cognition. Evol Anthropol 24:225-237.

Siegel JM (2005): Functional implications of sleep development. PLoS Biol 3:e178.

Siegel JM (2009): Sleep viewed as a state of adaptive inactivity. Nat Rev Neurosci 10:747-753.

Staunton H (2005): Mammalian sleep. Naturwissenschaften 92:203-220.

Stickgold R, Walker MP (2007): Sleep-dependent memory consolidation and reconsolidation. Sleep Med 8:331-343.

Xie L, Kang H, Xu Q, et al (2013): Sleep drives metabolite clearance from the adult brain. Science 342, 373-377. 